\title{
Conceptual Development of Hybrid Structures Towards Eco-Efficient Vehicle Lightweighting
}

\author{
Alexander Kaluza, Tim Fröhlich, Sebastian Kleemann, \\ Wolfgang Walk, Christoph Herrmann, Stephan Krinke \\ and Thomas Vietor
}

\begin{abstract}
Lightweight design is a major strategy in automotive development. The dominant motivation is a reduction of use-phase energy demands while retaining or improving technical performance. The application of new materials is the prevalent lightweighting strategy. Modern vehicle concepts extend material substitution up to the combination of different materials on a component level, so called hybrid designs. While engineering processes, methods and tools in design and production engineering are well established for conventional designs, hybrid designs pose new challenges. Lightweight materials as well as new manufacturing and recycling processes may cause increased environmental impacts. In order to achieve eco-efficient lightweight structures, energy savings from the vehicles' use phase need to compensate additional burdens in other lifecycle stages. The current work presents findings gained in a public-private research collaboration. Its starting point is the understanding of the role of life cycle engineering towards its impact on overall sustainability goals. Based on derived key requirements, an integrated life cycle engineering approach is developed. Activities and interfaces between life cycle engineering, component design and manufacturing are elaborated. A special focus is set on the conceptual design stage, as emerging materials and manufacturing technologies lead to a broader concept variety. This stage presents also a major lever for shaping the life cycle environmental impact of components.
\end{abstract}

\footnotetext{
A. Kaluza $(\bowtie) \cdot$ T. Fröhlich · S. Kleemann · W. Walk · C. Herrmann

S. Krinke · T. Vietor

Open Hybrid LabFactory e.V., 38440 Wolfsburg, Germany

e-mail: a.kaluza@tu-braunschweig.de
}
A. Kaluza · C. Herrmann
Chair of Sustainable Manufacturing and Life Cycle Engineering, Institute of Machine Tools and Production Technology (IWF),
Technische Universität Braunschweig, 38106 Brunswick, Germany
T. Fröhlich · S. Kleemann · T. Vietor
Institut for Engineering Design, Technische Universität Braunschweig, 38106 Brunswick, Germany
W. Walk · S. Krinke
Volkswagen AG, 38440 Wolfsburg, Germany
(C) The Author(s) 2018
E. Benetto et al. (eds.), Designing Sustainable Technologies,
Products and Policies, https://doi.org/10.1007/978-3-319-66981-6_21 


\section{Introduction}

Transport is accounting for a quarter of global greenhouse gas emissions with road transport being responsible for three thirds of this share. Towards increasing the environmental sustainability of the transport sector, use phase energy demands gained significant importance in the development of mass-produced vehicles. This is induced by legal requirements targeting greenhouse gas emissions for manufacturers' vehicle fleets. Several measures enable to improve the use phase efficiency, i.e. drivetrain adaptions or optimised aerodynamics. Introducing lightweight structures reduces energy demands during driving situations with frequent acceleration and deceleration as well as elevations. There are different strategies to achieve lightweight design. The predominant approach in vehicle engineering is material substitution. For instance, the application of high strength steels is widespread in the current vehicle generation. As those designs reach their limits in achieving further weight reductions, new approaches take the stage. While multi-material designs assign different materials to each component, hybrid designs combine different materials on a component level.

The integrated framework by Hauschild et al. is presented (see Fig. 1) in order to provide a perspective on the overall effect of lightweight structures regarding sustainability goals as well as the role of life cycle engineering (LCE) in this context [1]. Within the framework an environmental impact axis and a temporal axis span spaces of different scopes. Those scopes set a frame for separate optimisation with spatial/ geographical, organisational and technological implications. It is linked to the IPAT thinking that relates central driving forces to the production and consumption patterns in a future sustainable society. The total environmental impact is presented (I) as a function of the human population (P), the human affluence (A) and the technology factor $(\mathrm{T})$. While this represents a top-down approach within the above mentioned framework that is strongly interrelated with absolute sustainability limits, the publication on hand focuses on a complementary bottom-up approach. Here, product development plays a central role as a large share of the later environmental impact is already decided at this stage. Methods and tools should help to support decision making towards the upper scopes of concern, orienting it towards absolute sustainability. Within the framework, LCE is defined as sustainability-oriented product development activities within the scope of one to several product life cycles. The methods and tools used in LCE must support reducing the total environmental impact associated with technology change. Life cycle management (LCM) has to support this understanding on a company level [2]. As LCE activities relate to an integrated product and process life cycle planning, the interface between LCM and LCE is explained as a floating transition zone [3].

The overarching goal of the presented research is the understanding and adaption of engineering processes in conceptual design towards eco-efficient lightweight structures. The term eco-efficiency in general is defined according to [4] as environmental impact per unit of product. The current research is focusing on a rather technological perspective of that definition. Findings from executing development 


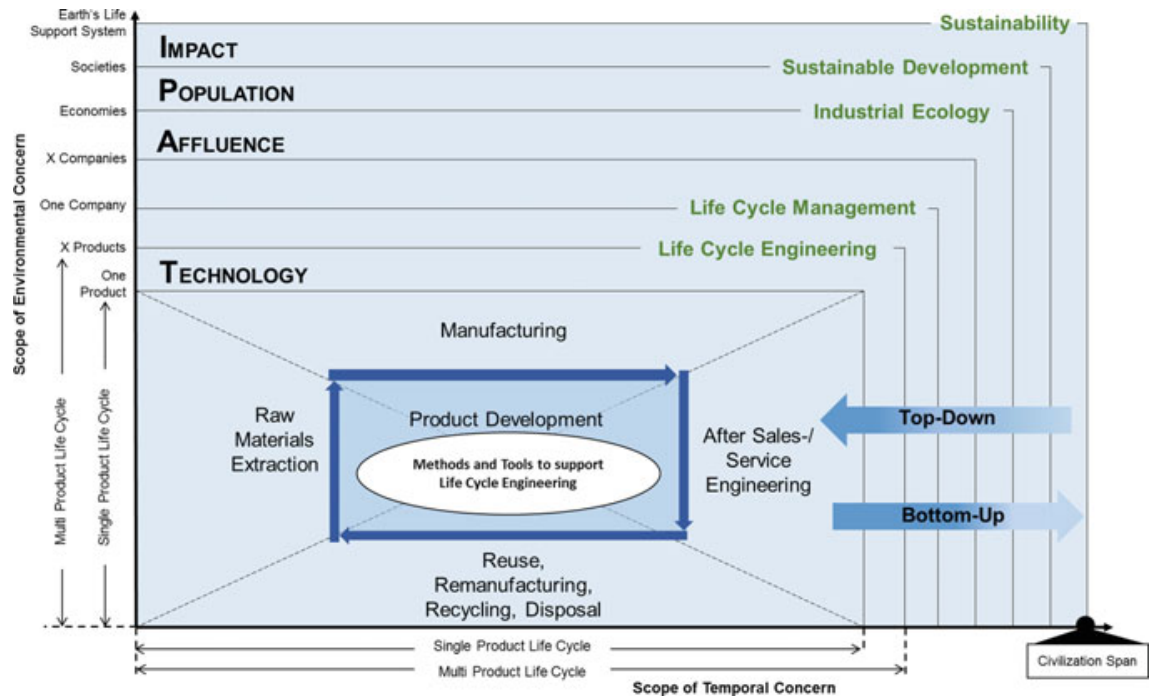

Fig. 1 Life Cycle Engineering (LCE) framework placing manufacturing within the context of planetary boundaries and absolute sustainability [1]

projects on lightweight structures in collaboration between research and large-scale industry production are reflected. This encompasses strengths and weaknesses of current exchange between the involved domains and the impacts of derived decisions. A generalization and aggregation to key requirements guides future research in LCE for lightweight structures. Further activities require to focus on the linkage of technology-based decisions towards a macro-level eco-efficiency enabling a broader sustainability performance, as e.g. promoted by Huppes \& Ishikawa [5].

\section{Automotive Lightweight Structures and LCE}

\subsection{Design of Automotive Lightweight Structures}

Lightweight design (LWD) is an engineering approach intending to reduce a product's weight without downgrading its performance. From a mechanical engineering point of view, the intention is to improve load-bearing capacities without increasing weight [6]. Lightweight design as a discipline is a combination of product development, material science as well as mechanics. Different lightweight strategies are distinguished. Material LWD promotes the substitution towards materials that show an improved technical performance for the specific application. Form LWD targets the shape and topology of structures according to the required load case. Manufacturing $L W D$ enables the manufacturing of lightweight structures through new processes. Constraint LWD subsumes external requirements that 
influence product weight, e.g. maximum payloads in civil engineering. Concept $L W D$ targets the reflection on component boundaries, e.g. aiming for functional integration [7].

LWD requires the joint consideration of all lightweighting strategies. The interplay between material selection and geometrical conceptualization is a major interface in LWD. Material selection based on material performance indicators, e.g. specific stiffness, shows major drawbacks. Those approaches often assume large cross-sections and thin-walled structures without any space restrictions. This is why the theoretical weight reduction numbers are hard to achieve in practice. In contrast to that, Wanner considered the boundary condition of limited installation space [8]. Additionally, automotive component development requires to strictly meet cost restrictions. Kleemann et al. as well as Fröhlich et al. discuss the dependencies of mechanical performance, weight and costs for an automotive roof structure [9]. The manufacturing of lightweight structures requires adaption of existing and qualification of new manufacturing processes, as summarized e.g. in [10]. Manufacturing routes that enable the realization of hybrid designs combine process technologies from the metals, plastics and textile industry. The integration of new processes in current process chains requires the development towards competitive cycle times, the qualification for sensitive process steps, e.g. the cathodic dip painting, as well as the consideration of additional invest volumes as exemplary shown in [11]. The variety and complexity of LWDs and manufacturing suggests to jointly elaborate the potentials and obstacles of a large-scale manufacturing from a design and manufacturing perspective.

\subsection{LCE for Automotive Lightweight Structures}

Several research papers discuss the topic of LCE in automotive engineering. Three constituting aspects of LCE are distinguished as derived from [12]. First, the need for an evaluation method, which enables to assess environmental impacts of products and processes, second the incorporation of a life cycle perspective and third the methods and tools required to achieve the consideration of those aspects in the engineering of products and processes. Life Cycle Assessment (LCA) according to ISO 14040 serves as an established evaluation method enabling the analysis and interpretation regarding different environmental impact categories. As illustrated in Fig. 2, the consideration of the entire product life cycle is crucial within the engineering of lightweight structures. Compared to conventional (steel) designs, lightweight structures often show higher environmental impacts from raw materials extraction. In order to enable eco-efficient LWDs on a product level, use phase energy savings need to compensate those burdens. In addition, potential efforts in the end-of-life phase occur, as conventional processes are not suitable for hybrid components and new processes lack maturity compared to the steel reference. Figure 2 schematically shows the influence of two lightweight alternatives on the environmental impacts of a vehicle component manufacturing in comparison to a 


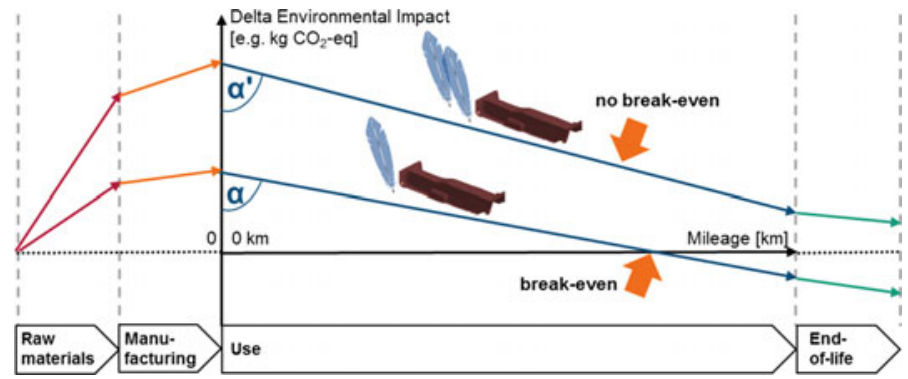

Fig. 2 Life cycle perspective of the influence of lightweight designs on vehicle environmental impacts. Relative representation compared to reference design (base line)

reference (y-intercept). The gradient (angle alpha; alpha') represents benefits due to a decreased energy consumption during use. The break-even point indicates the required driving distance to achieve an environmentally benign alternative. The end-of-life covers the effort to recover material, the avoided landfill and avoided primary material consumption due to the availability of secondary material. In the given example, two components are evaluated. One might reach the break-even during the predicted vehicle use, while the other lightweight alternative does not compensate additional burdens from raw materials and manufacturing during use.

Available methods and tools in LCE reach from qualitative tools to quantitative approaches [13]. Broch emphasises the importance of robust LCE decisions in automotive product development [14]. This includes considering parameters originating from the foreground and relevant background systems as described in [15]. The application of LCE methods and tools is widespread in industry-either taking a prospective or retrospective view. In automotive engineering, full LCA studies help to evaluate the impact of innovative technologies. Furthermore, the assessment of full vehicles is a common application. The integration of LCA results in product development is assisted through elaborated modelling tools. These enable an acceleration of the assessment. Detailed environmental evaluations are typically upstream and downstream activities. A detailed LCA in predevelopment would serve as an input for requirements definition. A downstream assessment of the final product ensures the meeting of requirements. In upstream application, challenges often occur in the adequate prediction of engineering parameters [16]. In order to enable LCE for the engineering with new materials, Ashby extends the property-driven material selection methodology through the consideration of eco-properties, e.g. the impact of raw materials extraction on climate change [17]. Shortcomings might occur as background system parameters are fixed to a certain extent. For example, regional effects in raw materials extraction might significantly influence resulting environmental impacts. Poulikidou et al. propose a method, which integrates design and life cycle assessment in automotive component development. The applied geometry retains for different material alternatives and only monolithic designs are discussed [18]. Lindner \& Schmitt provide a 
methodology that assists in the prediction of energy and resource flows for innovative composite manufacturing chains [19]. In summary, approaches covering single aspects within the LCE support of lightweight vehicle structures are present. However, a comprehensive methodology supporting the design and LCE of lightweight structures is missing for the automotive application. Experiences from aviation applications could serve as a fruitful input for the automotive case. However, the benefits of lightweight structures and consequently the environmental break-even differ due to longer use periods.

\section{Key Requirements for Effective LCE in the Development of Lightweight Concepts}

The current research builds on findings from a public-private collaboration comprising expertise from industrial practice and academic research. The joint development is executed in a lab environment that is designated for developing and providing methods and tools towards developing eco-efficient lightweight concepts for large-scale vehicle manufacturing. The engineering on a product and technology level is in the focus of the current research. This follows the bottom up approach of LCE as described in [1]. The collaboration between experts from design and production engineering as well as life cycle evaluation during the development of various hybrid vehicle lightweight structures enables the derivation of five key requirements. These build the basis for shaping methods \& tools that enable adequate LCE support on the technology level (see Fig. 3).

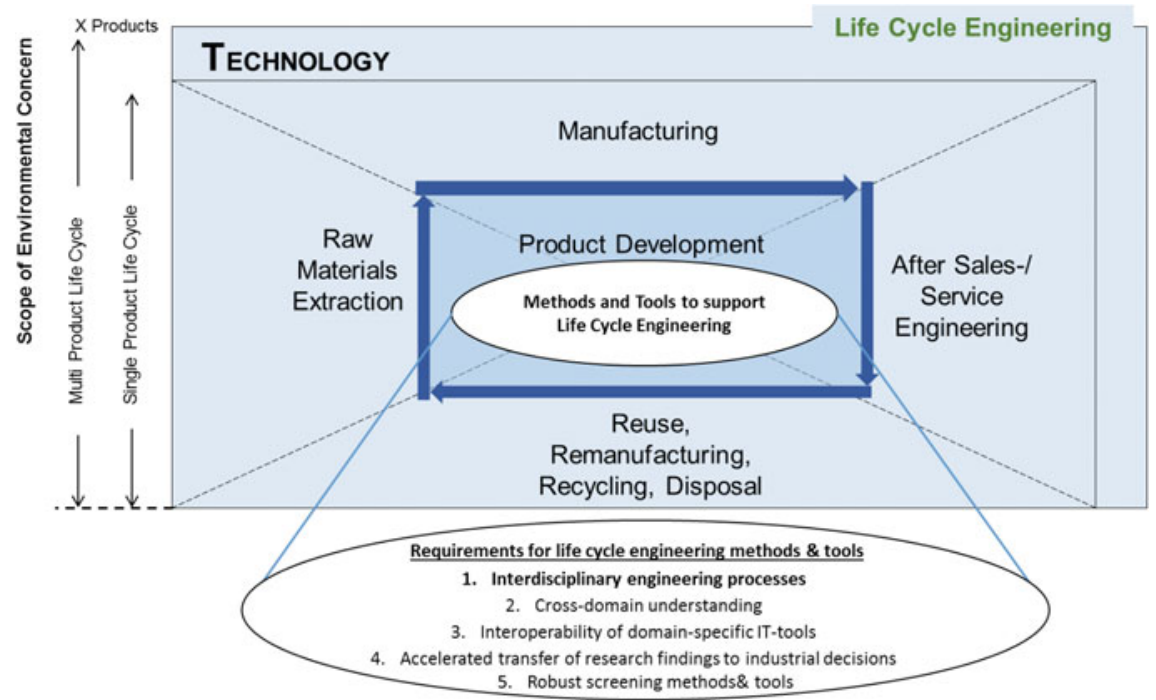

Fig. 3 Key requirements for methods and tools to support LCE of lightweight structures 
1. Interdisciplinary engineering processes: Concurrent engineering approaches are an established concept in the development of vehicle structures. When it comes to the design of hybrid or multi-material concepts, one has to face some specific challenges. Lightweight structures based on hybrid or multi-material approaches require new designs compared to established materials, comprehensive experience is currently missing. Manufacturing technologies for hybrid designs are developing rapidly with implications for parts' design and life cycle evaluation. A particularly high number of variations and gaps in LCA databases, e.g. manufacturing processes, disqualify click-and-rush LCA studies composed of standard modules. All of the considered engineering disciplines have to deal with high uncertainties and engineering processes that are even more dynamic than in conventional vehicle development. Especially product development and manufacturing technology development are to a certain extent parallel activities in the given case.

$2 \& 3$. Cross-domain understanding and interoperability of domain-specific IT tools: All domain-specific engineering activities require the assistance of advanced methods and tools, e.g. numerical simulation regarding crash behaviour or manufacturing process simulations. In addition, the modelling of energy and resource demands relies on complex models incorporating different data sources-from static life cycle inventories to the simulation of different use patterns or the consideration of live manufacturing data. Typically, simplified key performance indicators and visualization techniques are used to exchange information between disciplines. Their benefit lies in the easy interpretability. At the same time, the understanding for cross-disciplinary interlinkages decreases. As the decision scope shifts from the gate-to-gate perspective towards the whole product life cycle as well as across life cycles, domain-specific methods and tools should allow an information flow across disciplines.

4. Accelerated transfer of research findings to industrial decisions: In the context of lightweight structures, but similarly concerning technologies, innovation cycles become shorter including a dynamic innovation network. Thus, academic and industrial research to a certain extent parallel the development itself. Higher dynamics in the interaction of stakeholders are the result.

5. Robust screening methods \& tools for the acceleration of engineering processes: The introduction hybrid or multi-material lightweight structures significantly increases the solution space during the development of vehicle components. This leads to suspension of traditional geometries, component boundaries or installation spaces creating new challenges for methods and tools in the design domain. Cost assessment faces new challenges due to the fast changing designs and the evaluation of new materials, processes and geometries. For both environmental assessment and cost assessment, one major challenge is the scaling up of the applied models in order to predict the behaviour in vehicle mass production. This increased complexity in all disciplines, from engineering design over manufacturing planning up to LCE, needs to be reflected in developing evaluation tools that build on the described complexity, but enable to derive reliable decisions. 


\section{Interdisciplinary Engineering Processes}

In the following, an interdisciplinary engineering process for the conceptual development of lightweight vehicle structures is presented. This addresses the first key requirement as described in the previous section. The presented process extends prior work that focused on a functional modelling level [15]. Building on that, a holistic view on the conceptual development process of hybrid lightweight structures is in the focus of the current work. In this course, a detailed description of interfaces between component design, production engineering as well as life cycle evaluation is provided. Current component development processes consider the disciplines in various depths. Technical, economic and environmental performance are key targets in component development. While a constant tracking of the first two is established, environmental evaluation for new technologies is performed as upstream and downstream activity. The increasing complexity and concept variety through the introduction of lightweight structures, e.g. regarding their materials or joining technologies, suggests a constant tracking of environmental performance within the vehicle development process $[14,20]$. Each of the disciplines rely on own established methods and tailored tools. The proposed process interprets integrated LCE as a close and agile interlink between the disciplines (see Fig. 4).

Within the process, different columns represent the engineering disciplines, whereas four lanes describe the sequence of engineering activities in the respective

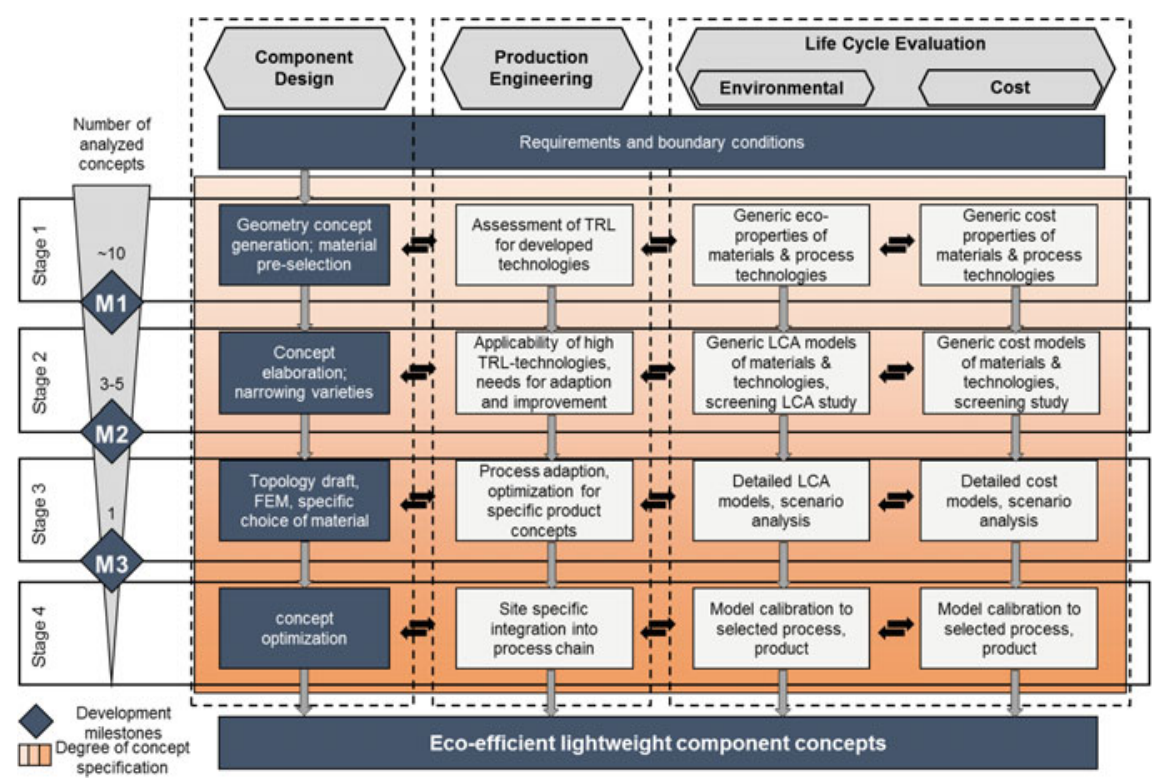

Fig. 4 Interaction between component design, production engineering and life cycle evaluation in an integrated LCE approach 
disciplines (stage 1 to stage 4). Based on a requirements definition by each of the disciplines, a generic component engineering process is followed. The component design provides the pace of the process (blue boxes). This complies with the established situation in vehicle manufacturers, where product development represents the key business process. Contrary to the current practice, production engineering and environmental life cycle evaluation are included as continuous and synergetic activities (white boxes). The sequence of engineering activities and interplays between the disciplines is broken down into sub-activities and interchanges. All activities on a horizontal level are executed as concurrent activities. At each stage, an interdisciplinary review is performed within defined milestones. This does not only result in a stepwise narrowing of uncertainties and the identification of interdisciplinary trade-offs, but also helps to sort out succeeding options while dropping other alternatives as represented by the funnel and respective milestones in Fig. 4. The main outcome of the engineering process are concepts for lightweight components that comply to the disciplines' requirements.

Stage 1 of the proposed process opens up a broad solution space for achieving the desired technical parameters through different geometries, materials and manufacturing routes. This stands in opposition to current component development processes, that rely on a strict breakdown of vehicle targets and thus limit variations for the developed concepts as described in [20]. The second stage represents a concept realisation with the assistance of analytic and simplified numeric calculations. As a result, further processing regards only a small number of concepts. The third stage covers numeric studies as measures to enable a detailed design with final specifications on material compositions as well as manufacturing routes for only one or few concepts. The fourth stage deals with the optimization of the concept in favour.

\section{Discussion and Outlook}

The introduction of hybrid lightweight structures poses new challenges on the support of LCE in automotive development. While the effect of lightweight structures on a broader sustainability perspective defines the overarching research demands, the scope of the current research focuses on a technology perspective. This enables to understand engineering activities and decisions on a micro-level to allow a further integration into a broader LCE context. An increasing variety of materials and manufacturing processes significantly widens the solution space in conceptual development of hybrid structures. In result, LCE methods \& tools need further integration side-by-side to engineering design and production engineering. Key requirements towards the adaption of LCE methods \& tools have been derived and the introduction of integrated engineering processes is elaborated. The approach targets the requirements in a double sense: Firstly, the process ensures that 
non-succeeding options are eliminated in early stages and efforts can be focused. Secondly, the process enables an evolutionary building on established methods and tools.

Further activities are required to enhance the capabilities of the presented approach. The elaboration of technical interfaces between domain-specific tools will accelerate the information exchange in the proposed manner. Coincidently, the identification of further interdisciplinary trade-offs will be enabled. Yet a small number of case studies have confirmed the presented approach. Within the public-private partnership Open Hybrid LabFactory further experience through implementation and adaption will be generated. The communication between disciplines could be enhanced by providing visualizations that enable a low barrier accessibility of the research results. One key facility at the Open Hybrid LabFactory is the newly established 'Life Cycle Design \& Engineering Lab', a workspace to explore modes of cross-discipline cooperation and visualisation techniques in order to support and promote integrated LCE.

Acknowledgements This research is funded by the German Federal Ministry of Education and Research (BMBF) within the research campus "Open Hybrid Lab Factory" and managed by the Project Management Agency Karlsruhe (PTKA). The authors are responsible for the contents of this publication.

\section{References}

1. Hauschild M. Z, Herrmann C, Kara S, An Integrated Framework for Life Cycle Engineering. Procedia CIRP, 61, 2-9, 2017, https://doi.org/10.1016/j.procir.2016.11.257.

2. Herrmann C, Ganzheitliches Life Cycle Management. SpringerVerlag Berlin Heidelberg, 2010, https://doi.org/10.1007/978-3-642-01421-5.

3. Umeda Y, Takata S, Kimura F, Tomiyama T, Sutherland J.W, Kara S, Duflou J. R, Toward integrated product and process life cycle planning-An environmental perspective. CIRP Annals-Manufacturing Technology, 61(2), 681-702, 2012, https://doi.org/10.1016/j.cirp. 2012.05.004.

4. Huppes G, Ishikawa M, A framework for quantified eco-efficiency analysis. Journal of industrial ecology, 9(4), 25-41, 2005, https://doi.org/10.1162/108819805775247882.

5. Huppes G, Ishikawa M, Eco-efficiency guiding micro-level actions towards sustainability: Ten basic steps for analysis. Ecological Economics, 68(6), 1687-1700, 2009, https://doi.org/ 10.1016/j.ecolecon.2009.01.007.

6. Wiedemann J, Leichtbau. Berlin, Heidelberg: Springer Berlin Heidelberg, 2007, https://doi. org/10.1007/978-3-540-33657-0.

7. Friedrich H.E, Leichtbau in der Fahrzeugtechnik, 2013, https://doi.org/10.1007/978-3-83482110-2.

8. Wanner A, Minimum-weight materials selection for limited available space. Materials \& Design, 31(6), 2834-2839, 2010, https://doi.org/10.1016/j.matdes.2009.12.052.

9. Kleemann S, Fröhlich T, Türck E, Vietor T, A Methodological Approach Towards Multi-material Design of Automotive Components. Procedia CIRP, 60, 68-73, 2017, https:// doi.org/10.1016/j.procir.2017.01.010. 
10. Taub A.I, Luo A.A, Advanced lightweight materials and manufacturing processes for automotive applications. MRS Bulletin, 40(12), 1045-1054, 2015, https://doi.org/10.1557/ mrs.2015.268.

11. Eckstein L, Ickert L, Goede M, Dölle N, Lightweight Floor Structure with Reinforcements of CFRP and GFRP. ATZ Autotechnology, 11(2), 48-52, 2011.

12. Jeswiet J, Life Cycle Engineering. In CIRP Encyclopedia of Production Engineering (pp. 757-758), Berlin, Heidelberg: Springer Berlin Heidelberg, 2014, https://doi.org/10.1007/ 978-3-642-20617-7_6609.

13. Bovea M.D, Pérez-Belis V, A taxonomy of ecodesign tools for integrating environmental requirements into the product design process. Journal of Cleaner Production, 20(1), 61-71, 2012. https://doi.org/10.1016/j.jclepro.2011.07.012.

14. Broch F, Warsen J, Krinke S, Implementing Life Cycle Engineering in Automotive Development as a Helpful Management Tool to Support Design for Environment. In G. Sonnemann \& M. Margni (Eds.), Life Cycle Management (pp. 319-329). Springer, 2015, https://doi.org/10.1007/978-94-017-7221-1.

15. Kaluza A, Kleemann S, Fröhlich T, Herrmann C, Vietor T, Concurrent Design \& Life Cycle Engineering in Automotive Lightweight Component Development. Procedia CIRP, 66, 16-21, 2017, https://doi.org/10.1016/j.procir.2017.03.293.

16. Broch F, Integration von ökologischen Lebenswegbewertungen in Fahrzeugentwicklungsprozesse. Springer, 2017.

17. Ashby M.F, Introduction. In Materials Selection in Mechanical Design (pp. 1-13). Elsevier, 2011, https://doi.org/10.1016/b978-1-85617-663-7.00001-1.

18. Poulikidou S, Schneider C, Björklund A, Kazemahvazi S, Wennhage P, Zenkert D, A material selection approach to evaluate material substitution for minimizing the life cycle environmental impact of vehicles. Materials \& Design, 83, 704-712, 2015, https://doi.org/10. 1016/j.matdes.2015.06.079.

19. Lindner F, Schmitt R, Goal-oriented life cycle investigations for composite manufacturing chains. Procedia CIRP, 29, 438-443, 2015, https://doi.org/10.1016/j.procir.2015.02.043.

20. Kaluza A, Kleemann S, Broch F, Herrmann C, Vietor T, Analyzing decision-making in automotive design towards life cycle engineering for hybrid lightweight components. Procedia CIRP, 50, 825-830, 2016, https://doi.org/10.1016/j.procir.2016.05.029.

Open Access This chapter is licensed under the terms of the Creative Commons Attribution 4.0 International License (http://creativecommons.org/licenses/by/4.0/), which permits use, sharing, adaptation, distribution and reproduction in any medium or format, as long as you give appropriate credit to the original author(s) and the source, provide a link to the Creative Commons license and indicate if changes were made.

The images or other third party material in this chapter are included in the chapter's Creative Commons license, unless indicated otherwise in a credit line to the material. If material is not included in the chapter's Creative Commons license and your intended use is not permitted by statutory regulation or exceeds the permitted use, you will need to obtain permission directly from the copyright holder.

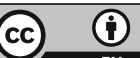

